\title{
Motion properties of planar two-degree-of-freedom mechanisms
}

\author{
K.N. Umesh ${ }^{a}$, C. Amarnath b,* \\ ${ }^{a}$ Mandya Engineering College, Mandya, Karnataka, India \\ ${ }^{\mathrm{b}}$ Department of Mechanical Engineering, Indian Institute of Technology, Mumbai 400076, India
}

\begin{abstract}
A technique for analysis of second order properties of planar two-degree-of-freedom mechanisms has been proposed. Analytical and graphical representations of the properties have been used to study the relative influence of input velocities and accelerations on the acceleration of the end point. It has been shown that best control on end point acceleration is available at and in the vicinity of the isotropic point.
\end{abstract}

\section{Introduction}

Increased adoption of multi-degrees-of-freedom motions in robotic devices and machinery has resulted in extensive studies on the properties of such systems. Measures such as volume of workspace, reach, mobility of input links have been developed to illustrate geometric properties [1-3]. Motion properties of the mechanisms have been examined and attempts have been made to quantify and characterize motion performance by developing performance evaluation indices. Force error analysis has been addressed by Salisbury and Roth [4] and Asada and Ro [5]. Yashikawa [6] has introduced the concept of manipulability, while Ghosal and Roth [7] propose quantities based on transmission efficiency defined in terms of areas and volumes. Lee et al. [8], propose several measures to evaluate kinematic cross coupling, directional motion mobility, force manipulability and motion efficiency.

Studies on motion properties have generally been restricted to the so called local properties at a given position of the mechanism. One result of such studies is the identification of isotropic points from a study of the first order properties and it has been suggested that the workplace in the 
proximity of these points is ideally suited for grasp and manipulation since the end point has uniform mobility in all directions [4]. In discussing this mobility most authors refer to the velocity ellipse, which could be considered as a graphical representation of the Jacobian matrix. Asada and Ro [5], and Lee et al. [8] propose the use of a distribution map of these ellipses in the workspace of a manipulator to evaluate the motion performance as a whole. Howe et al. [9] and Ou and Tsai [10] have conducted a similar exercise and have plotted the condition number, their aim being the identification of a portion of the workspace close to the isotropic point.

Main body motions in many robotic manipulators are obtained from planar two-degrees-offreedom mechanisms. Hence in this paper an attempt has been made to examine the local second order properties of such devices and arrive at graphical representations similar to the velocity ellipse. These graphical representations permit one to examine the relative influence of input rates and input accelerations on the acceleration of the end point. It has been determined that the isotropic point is of importance when one considers the second order motion properties also.

\section{Instantaneous motion properties}

The path generated by a point on a moving link in a $m$-dimensional task space is a function of $n$ independent parameters. As our study is confined to planar 2DOF linkages, we have in this case $m=n=2$.

The position of the end point in a fixed reference frame can be related to the motion parameters by

$$
\mathbf{p}=\left\{p_{x}, p_{y}\right\}^{\mathrm{T}}
$$

where, $p_{x}=f\left(\theta_{1}, \theta_{2}\right), p_{y}=g\left(\theta_{1}, \theta_{2}\right)$ and $\boldsymbol{\Theta}$ is a $(n \times 1)$ vector representing the joint variables, $\theta_{1}, \theta_{2}$.

\subsection{First order properties}

The relationship between joint rates $\dot{\boldsymbol{\Theta}}$ and the velocity of the moving point is obtained by differentiating Eq. (1) with respect to time and is given by

$$
v=\mathbf{J} \dot{\Theta}
$$

where,

$$
\mathbf{J}=\left[\begin{array}{ll}
\frac{\partial f}{\partial \theta_{1}} & \frac{\partial f}{\partial \theta_{1}} \\
\frac{\partial g}{\partial \theta_{1}} & \frac{\partial g}{\partial \theta_{2}}
\end{array}\right]
$$

One can vary $\dot{\boldsymbol{\Theta}}$ to obtain the distribution of velocities of the end point at a given position. To do so it is meaningful to consider some constraint on the inputs $\dot{\boldsymbol{\Theta}}$. Several authors [5,7,11] have used normalizing constraints like quadratic relations of the form, $\dot{\boldsymbol{\Theta}}^{\mathrm{T}} \dot{\boldsymbol{\Theta}}=k^{2}$ and have determined that the locus of the tips of velocity vectors $v$ is an ellipse. The ovality of the ellipse is an indication of the relative motion possibilities in various directions and the condition number of $\mathbf{J}$ could be 
used as an indicator of the ovality of the ellipse. Close to the boundary of the workspace, the ellipse is long and narrow and the end point cannot move in all directions with equal ease. At an isotropic configuration the ellipse reduces to a circle and one can obtain uniform mobility in all directions. At other points in the workspace, the ease of motion depends on the ovality of the ellipse. The mobility is fairly uniform in a portion of the workspace in the vicinity of the isotropic point and this zone could be deemed to be ideal for performing tasks like grasping and manipulation [4].

\subsection{Second order properties}

Having reviewed the first order properties we now examine the second order properties. To characterize these properties Ghosal and Roth [7] propose the use of a scalar quantity, which is proportional to the area of the velocity ellipse. In what follows we adopt a different approach.

The acceleration $\mathbf{f}$ of the moving point obtained by differentiating Eq. (2) with respect to time is given by

$$
\mathbf{f}=\mathbf{f}_{j}+\mathbf{f}_{q}
$$

where, $\mathbf{f}=\left\{f_{x}, f_{y}\right\}^{\mathrm{T}}$ is the total acceleration of the end point. Vector $\mathbf{f}_{j}$ is linear in $\ddot{\boldsymbol{\Theta}}$ while $\mathbf{f}_{q}$ is nonlinear in $\dot{\boldsymbol{\Theta}}$. Vector $\mathbf{f}_{j}=\mathbf{J} \ddot{\boldsymbol{\Theta}}$ where $\ddot{\boldsymbol{\Theta}}$ is the input angular acceleration vector and $\mathbf{f}_{\mathbf{q}}=\left\{q_{x}, q_{y}\right\}^{\mathrm{T}}$ is a vector of quadratic forms. The quadratic form corresponding to $f_{x}$ is $q_{x}=\dot{\boldsymbol{\Theta}}^{\mathrm{T}} \mathbf{A} \dot{\boldsymbol{\Theta}}$ and $q_{y}=\dot{\boldsymbol{\Theta}}^{\mathrm{T}} \mathbf{B} \dot{\boldsymbol{\Theta}}$ is the quadratic form corresponding to $f_{y}$ [12]. The coefficient matrix $\mathbf{A}$ of quadratic form $q_{x}$, is given by

$$
\mathbf{A}=\left[\begin{array}{cc}
\frac{\partial^{2} f}{\partial \theta_{1}^{2}} & \frac{\partial^{2} f}{\partial \theta_{1} \partial \theta_{2}} \\
\frac{\partial^{2} f}{\partial \theta_{1} \partial \theta_{2}} & \frac{\partial^{2} f}{\partial \theta_{2}^{2}}
\end{array}\right]
$$

The coefficient matrix $\mathbf{B}$ of quadratic form $q_{y}$ is similar to $\mathbf{A}$ and is obtained by using the partial derivatives of $g$ instead of $f$. Both the coefficient matrices $\mathbf{A}$ and $\mathbf{B}$ are symmetric but as such nothing can be said about their definiteness [12].

We shall now study the acceleration distribution first by setting $\dot{\boldsymbol{\Theta}}$ to zero and imposing normalizing constraint on $\ddot{\boldsymbol{\Theta}}$. In the second instance we set $\ddot{\boldsymbol{\Theta}}$ equal to zero and impose a normalizing constraint on $\dot{\boldsymbol{\Theta}}$.

Case 1: $\dot{\boldsymbol{\Theta}}=\mathbf{0}$

With $\dot{\boldsymbol{\Theta}}$ equal to zero the end point has zero velocity, a situation which occurs when the mechanism is starting its motion from rest. Since $\dot{\boldsymbol{\Theta}}=\mathbf{0}, \mathbf{f}_{q}$ vanishes and we have $\mathbf{f}=\mathbf{J} \ddot{\Theta}$. The Jacobian $\mathbf{J}$ governs the distribution of acceleration and with a normalizing constraint in the form $\ddot{\boldsymbol{\Theta}}^{\mathrm{T}} \ddot{\boldsymbol{\Theta}}=k_{j}^{2}$ imposed on $\ddot{\boldsymbol{\Theta}}$, we obtain an ellipse, ellipse A in Fig. 1, similar to the velocity ellipse discussed earlier. The center of the ellipse coincides with the origin of all the acceleration vectors. It is obvious that under these conditions the acceleration distribution is along lines similar to the velocity distribution and we thus note that accelerating the end point, from rest, with equal ease in all directions is possible at an isotropic configuration. 

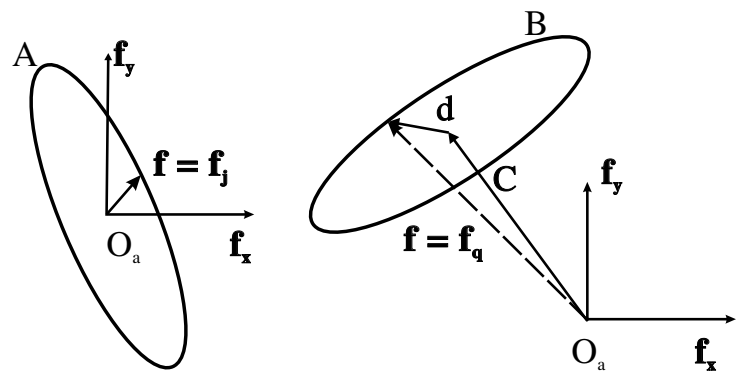

Fig. 1. Ellipses A and B.

When $\dot{\boldsymbol{\Theta}}$ is set to a prescribed nonzero value, we have in addition a constant acceleration vector $\mathbf{f}_{q}$ and the center of ellipse $\mathrm{A}$ is at the tip of $\mathbf{f}_{q}$.

Case 2: $\ddot{\boldsymbol{\Theta}}=\mathbf{0}$

We now consider case wherein $\ddot{\boldsymbol{\Theta}}=\mathbf{0}$ and impose a normalizing constraint on $\dot{\boldsymbol{\Theta}}$. With $\dot{\boldsymbol{\Theta}}$ constrained to $\dot{\boldsymbol{\Theta}}^{\mathrm{T}} \dot{\boldsymbol{\Theta}}=k_{q}^{2}, \mathbf{f}_{q}$ is a sum of two vectors.

$$
\mathbf{f}_{q}=\mathbf{c}+\mathbf{d}
$$

The magnitudes of the vectors $\mathbf{c}$ and $\mathbf{d}$ vary with the square of $k_{q}$. Further the expressions for the vectors $\mathbf{c}$ and $\mathbf{d}$ can be obtained by setting up a parametric representation of the constraint $\dot{\theta}_{1}^{2}+\dot{\theta}_{2}^{2}=k_{q}^{2}$ (a circle) as $\dot{\theta}_{1} k_{q} \cos (\psi)$ and $\dot{\theta}_{2} k_{q} \sin (\psi)$. Vector $\mathbf{c}$ is a constant, whose elements are given by

$$
c_{1}=k_{q}^{2}\left(a_{11}+a_{22}\right) / 2 \text { and } c_{2}=k_{q}^{2}\left(b_{11}+b_{22}\right) / 2
$$

where $a_{i j}$ and $b_{i j}$ are the elements of the coefficient matrices $\mathbf{A}$ and $\mathbf{B}$ respectively,

Vector $\mathbf{d}$ is given by

$$
\mathbf{d}=k_{q} L \boldsymbol{\Phi}
$$

where, $\mathbf{L}$ is a linear transformation matrix, whose elements are

$$
\begin{array}{ll}
l_{11}=\left(a_{11}-a_{22}\right) / 2 ; & l_{12}=a_{12} \\
l_{21}=\left(b_{11}-b_{22}\right) / 2 ; & l_{22}=b_{12}
\end{array}
$$

and $\boldsymbol{\Phi}=\left\{\phi_{1} \phi_{2}\right\}^{\mathrm{T}}$ is a vector with elements, $\phi_{1}=k_{q} \cos (2 \psi)$ and $\phi_{2}=k_{q} \sin (2 \psi)$.

The transformation matrix $\mathbf{L}$ and the constant vector $\mathbf{c}$ are position dependent and they are functions of link parameters $l_{1}$ and $\theta_{1}$. With $\dot{\boldsymbol{\Theta}}$ constrained, the locus of tip of vector $\mathbf{d}$ is an ellipse (ellipse B in Fig. 1) which is centered at the tip of constant vector c. The size of the ellipse is determined by the eigenvalues $\lambda_{1}$ of the matrix $\left[\mathbf{L} \mathbf{L}^{\mathrm{T}}\right]^{-1}$ and the constant $k_{q}$. The orientation is given by the eigenvectors $\mathbf{c}_{1}$ of $\left[\mathbf{L L}^{\mathrm{T}}\right]^{-1}$ and the semi-major axis $a=k_{q}^{2} / \sqrt{\lambda_{1}}$ and minor axis $b=k_{q}^{2} / \sqrt{\lambda_{2}}$, when say $\lambda_{1}<\lambda_{2}$. The orientation of the major axis of the ellipse $\mathrm{B}$ and the orientation of $\mathbf{f}_{q}$ are independent of the magnitudes of $\ddot{\boldsymbol{\Theta}}$. When $\ddot{\boldsymbol{\Theta}}$ is set to $\mathbf{0}, \mathbf{f}_{j}$ equals $\mathbf{0}$ and the origin of vector $\mathbf{c}$ coincides with the origin of the total acceleration vectors. When $\ddot{\boldsymbol{\Theta}}$ is set equal to a 


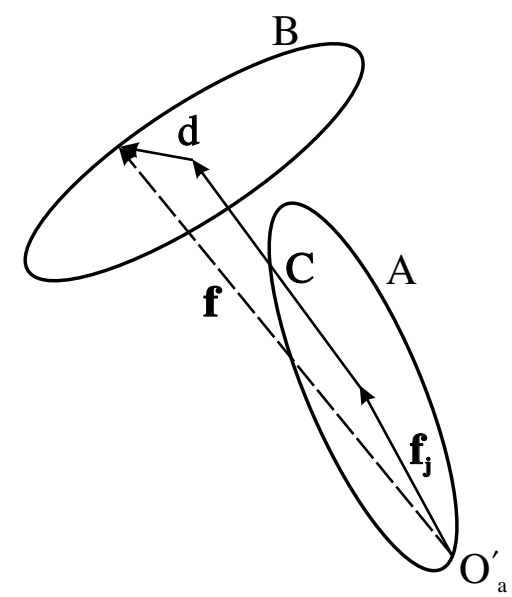

Fig. 2. Influence of motor rates and accelerations.

prescribed nonzero value, the total acceleration is given by $\mathbf{f}_{j}+\mathbf{c}+\mathbf{d}$. When $\mathbf{L}$ becomes singular the ellipse B degenerates to a straight line. The singularity of $\mathbf{L}$ is not related to the singular position of the manipulator.

\section{Case 3: Normalized constraints on $\dot{\boldsymbol{\Theta}}$ and $\ddot{\boldsymbol{\Theta}}$}

We now examine the acceleration distribution when normalizing constraints are imposed on both $\dot{\boldsymbol{\Theta}}$ and $\ddot{\boldsymbol{\Theta}}$. To do so we superimpose the results obtained by setting $\dot{\boldsymbol{\Theta}}=\mathbf{0}$ and varying $\ddot{\boldsymbol{\Theta}}$ in form $\ddot{\boldsymbol{\Theta}}^{\mathrm{T}} \ddot{\boldsymbol{\Theta}}=k_{j}^{2}$ on those obtained by setting $\ddot{\boldsymbol{\Theta}}=\mathbf{0}$ and varying $\dot{\boldsymbol{\Theta}}$ in the form $\dot{\boldsymbol{\Theta}}^{\mathrm{T}} \dot{\boldsymbol{\Theta}}=k_{q}^{2}$.

One such superposition is shown in Fig. 2. In measuring the acceleration vector $\mathbf{f}, \mathbf{f}_{j}, \mathbf{c}$ and $\mathbf{d}$ as shown, we imply that the origin $O_{\mathrm{a}}^{\prime}$ of the total acceleration vector $\mathbf{f}$ lies on the periphery of ellipse A. The representation in Fig. 2 enables us to study the influence of $\ddot{\boldsymbol{\Theta}}$ on the acceleration of the end point.

When ellipse A encompasses ellipse B the implication is that $\ddot{\Theta}$ has a relatively larger influence on end point acceleration than $\dot{\boldsymbol{\Theta}}$ has. On the other hand when the ellipse B is totally outside ellipse A the implication is that $\dot{\boldsymbol{\Theta}}$ has a greater influence on end point acceleration. Since $\dot{\boldsymbol{\Theta}}$ and $\ddot{\boldsymbol{\Theta}}$ represent in some form the velocities and accelerations of the actuators the above depicts the relative influences of motor velocities and accelerations on the acceleration of the end point along its trajectory.

\subsection{Isotropy}

At an isotropic position we have a circle instead of the ellipse A. In Fig. 3, a situation wherein ellipse B is totally inside the circle is shown. The motor acceleration capabilities are best utilized in this situation as motor accelerations have a dominating influence in all directions. This influence increases as motor velocities reduce to zero and ellipse B and vector $\mathbf{c}$ shrink as magnitude of end point velocity reduces. We could thus state that an end point, at rest at an isotropic position, can accelerate in all directions with equal ease. 


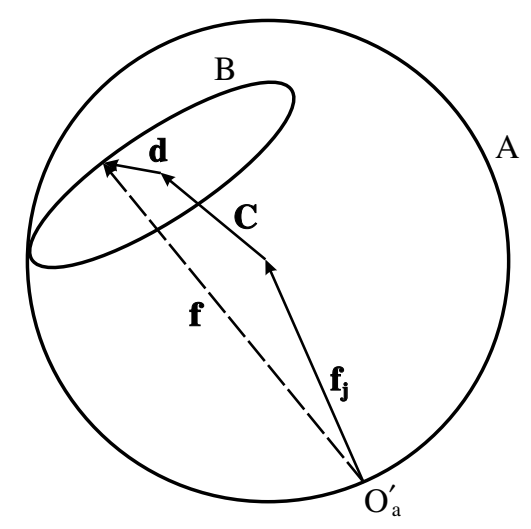

Fig. 3. Behavior at isotropic position.

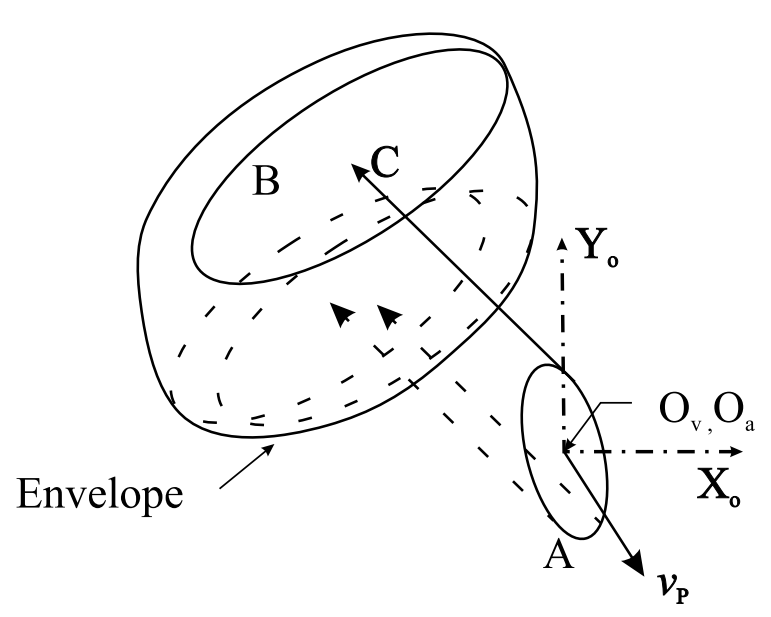

Fig. 4. Acceleration capabilities.

The implications of using motors with low acceleration capability could be seen in Fig. 4. In Fig. 4 ellipse A corresponding to $\ddot{\boldsymbol{\Theta}}^{\mathrm{T}} \ddot{\boldsymbol{\Theta}}=k_{j}^{2}$ and $\dot{\boldsymbol{\Theta}}=\mathbf{0}$, has its center at $O_{\mathrm{a}}$, where $O_{\mathrm{a}}$ is the origin of vector $\mathbf{f}_{j}$. The tip of $\mathbf{f}_{j}$ moves around the periphery of ellipse A as $\ddot{\boldsymbol{\Theta}}$ is varied. We add the vector $\mathbf{c}$ (and ellipse $\mathrm{B}$ ) to $\mathbf{f}_{j}$ to obtain total acceleration $\mathbf{f}$. In doing so, we have numerous ellipses $\mathrm{B}$ and associated vectors $\mathbf{c}$ positioned around ellipse A, with origin of vector $\mathbf{c}$ lying on periphery of ellipse A. The addition implies that vectors $\mathbf{c}$ and associated ellipses B must always remain parallel to themselves as origin of vector $\mathbf{c}$ moves around periphery of ellipse A. The set of ellipses B so obtained will have an outer envelope as shown in Fig. 4. With both $\dot{\boldsymbol{\Theta}}$ and $\ddot{\Theta}$ quadratically constrained, tips of all possible total accelerations $\mathbf{f}$ of the end point lie within this outer envelope.

When motors have poor acceleration capabilities and motor rates are high (resulting in increased end point velocities), ellipse A shrinks and the outer envelope of ellipses B could lie on one side of the origin $O_{\mathrm{a}}$ as shown in Fig. 4. Let us now consider an end point velocity $v_{\mathrm{p}}$ as shown in Fig. 4, the origin of vector $v_{\mathrm{p}}$ being $O_{\mathrm{v}}$ coincident with $O_{\mathrm{a}}$. Then with velocity vector $v_{\mathrm{p}}$ as shown and because 
all possible total acceleration vectors lie on one side of $O_{\mathrm{a}}$, one could only decelerate the end point along its path and not accelerate it. The reverse is possible when end point velocity is $\left(-v_{\mathrm{p}}\right)$.

\section{Illustrative examples}

In this section we examine the behavior of two planar mechanisms, a $2 R$ manipulator, which is an open chain, and a 5-bar manipulator a closed chain (Fig. 5). One could study the behavior of these linkages by imposing a quadratic constraint on the inputs. However, we impose constraints of the form $-p_{i} \leqslant \dot{\theta}_{i} \leqslant+p_{i}$ and $-q_{i} \leqslant \ddot{\theta}_{i} \leqslant+q_{i}$, rather than normalizing constraints since these constraints are close to those encountered in practice. Geometrically these constraints would represent rectangles in the $\left(\dot{\theta}_{1}, \dot{\theta}_{2}\right)$ spaces.

\subsection{R Manipulator}

We shall study the motion of the end point $\mathrm{B}$ of a $2 R$ manipulator linkage as shown in Figs. 5 and 6. The elements of Jacobian $\mathbf{J}$ and the matrices $\mathbf{A}$ and $\mathbf{B}$ are given in Appendix A. We consider the case $l_{1}=1.0, l_{2}=0.75, \theta_{1}=0^{\circ}$ and $\theta_{2}=30^{\circ}$, a nonsingular configuration and the
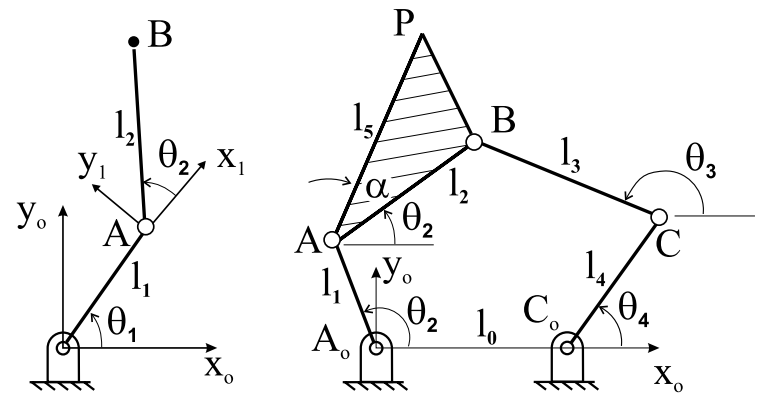

Fig. 5. 2R manipulator and 5-bar linkage.

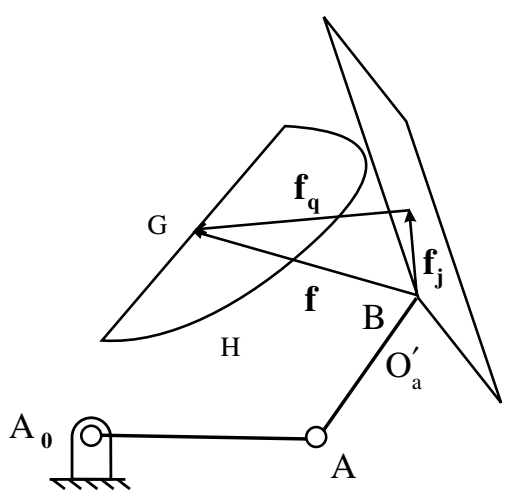

Fig. 6. Influence of motor rates and accelerations $-2 R$ manipulator. 
constraints on inputs are $-p_{i} \leqslant \dot{\theta}_{i} \leqslant+p_{i}$ and $-q_{i} \leqslant \ddot{\theta}_{i} \leqslant+q_{i}$. A rectangle in the $\left(\dot{\theta}_{1}, \dot{\theta}_{2}\right)$ space is mapped into a parallelogram in the $\left(v_{x}, v_{y}\right)$ space. With $\dot{\boldsymbol{\Theta}}=\mathbf{0}$, a rectangle in the $\left(\ddot{\theta}_{1}, \ddot{\theta}_{2}\right)$ space is also mapped into a parallelogram in $\left(f_{x}, f_{y}\right)$ space as shown in Fig. 6. With $\ddot{\Theta}$ set to zero and $\dot{\boldsymbol{\Theta}}$ constrained to a rectangle in the $\left(\dot{\theta}_{1}, \dot{\theta}_{2}\right)$ space we obtain a closed figure in the $\left(f_{x}, f_{y}\right)$ space as shown in Fig. 6. This figure is composed of two segments $G$ and $H . G$ is a straight-line corresponding to $-1.0 \leqslant \dot{\theta}_{2} \leqslant+1.0$ and $\dot{\theta}_{2}= \pm 1.5$. $H$ is a curve, corresponding to $-1.5 \leqslant \dot{\theta}_{1} \leqslant+1.5$ and $\dot{\theta}_{2}= \pm 1.0$, since normal and Coriolis accelerations are involved in this instance [13].

\subsubsection{Planar 5-bar manipulator}

The linkage with its end point at $P$ is shown in Figs. 5 and 7. In Fig. $5, l_{i}$ is the length of the $i$ th link and $\theta_{i}$ is the absolute angular position of $i$ th link measured w.r.t. positive $X_{0}$ axis. The elements of the Jacobian, $\mathbf{J}$ and coefficient matrices, $\mathbf{A}$ and $\mathbf{B}$ are given in Appendix A.

The end point $P$ and the associated 5-bar linkage $\left(l_{0}=1.1, l_{1}=1.0, l_{2}=0.875, l_{3}=0.75\right.$, $\left.l_{4}=0.713, l_{5}=1.125\right)$ is at an isotropic configuration $\left(\theta_{1}=84^{\circ}, \theta_{2}=23.118^{\circ}, \theta_{3}=120.024^{\circ}\right.$, $\left.\theta_{4}=75^{\circ}\right)$. A square in $\left(\ddot{\theta}_{1}, \ddot{\theta}_{4}\right)$ space is mapped into similar square in $\left(f_{x}, f_{y}\right)$ space when the input accelerations are varied as $-9.0 \leqslant \ddot{\theta}_{1} \leqslant+9.0$. With $\ddot{\boldsymbol{\Theta}}=\mathbf{0}$ and the motor rates varied as $-1.5 \leqslant \dot{\theta}_{1} \leqslant+1.5,\left(\dot{\theta}_{2}= \pm 1.0\right)$ and $-1.0 \leqslant \dot{\theta}_{2} \leqslant+1.0\left(\dot{\theta}_{1}= \pm 1.5\right)$ a rectangle in the $\left(\dot{\theta}_{1}, \dot{\theta}_{2}\right)$ space is mapped into a closed figure composed of two intersecting curves $G$ and $H$ as shown in Fig. 7 [13].

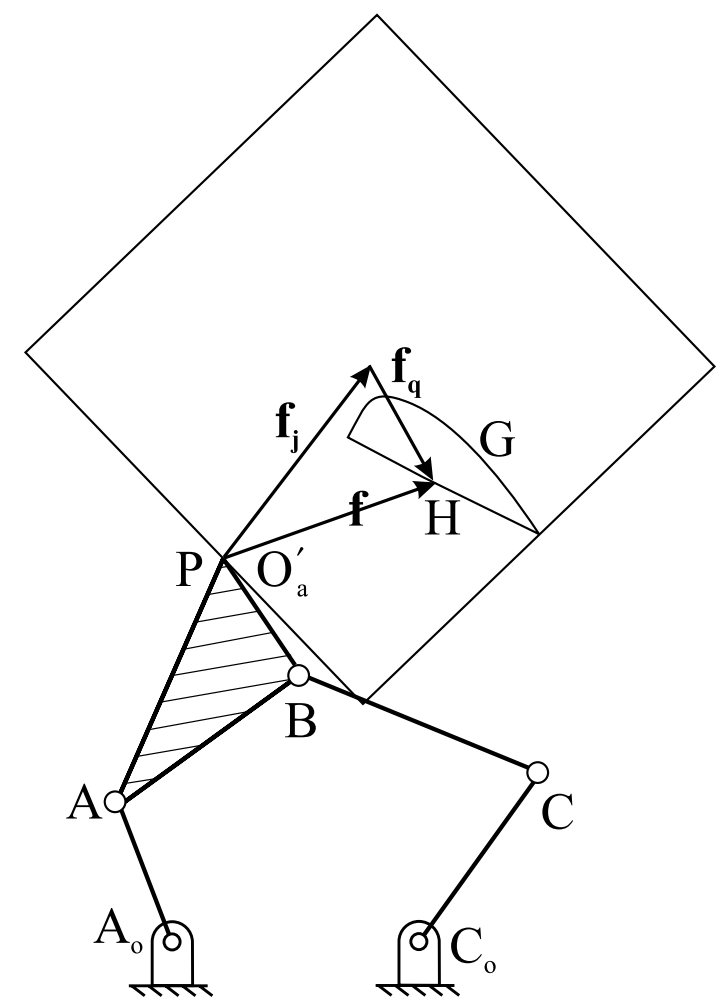

Fig. 7. Illustration of influence of motor rates and accelerations in 5-bar linkage. 


\section{Conclusions}

It has been shown that in planar two-degree-of-freedom linkages:

(a) One could obtain some insight into the acceleration capabilities of an end effector by imposing normalizing constraints on motor velocities and accelerations.

(b) An end effector at rest at an isotropic point can accelerate in all directions with equal ease.

\section{Acknowledgement}

The authors are thankful to their colleague Prof. K.K. Issac for suggestions and discussions.

\section{Appendix A}

\section{A.1. 2R Linkage}

The elements of the Jacobian $\mathbf{J}$ of the end point B of a $2 R$ linkage (Fig. 5) are given by

$$
\begin{aligned}
& j_{11}=-l_{1} S \theta_{1}-l_{2} S\left(\theta_{1}+\theta_{2}\right) \\
& j_{12}=-l_{2} S\left(\theta_{1}+\theta_{2}\right) \\
& j_{21}=+l_{1} C \theta_{1}+l_{2} C\left(\theta_{1}+\theta_{2}\right) \\
& j_{22}=+l_{2} C\left(\theta_{1}+\theta_{2}\right)
\end{aligned}
$$

And the elements of the coefficient matrices $\mathbf{A}$ and $\mathbf{B}$ are

$$
\begin{aligned}
& a_{11}=-l_{1} C \theta_{1}-l_{2} C\left(\theta_{1}+\theta_{2}\right) \\
& a_{12}=a_{21}=-l_{2} C\left(\theta_{1}+\theta_{2}\right), \\
& a_{22}=-l_{2} C\left(\theta_{1}+\theta_{2}\right), \\
& b_{11}=-l_{1} S \theta_{1}-l_{2} S\left(\theta_{1}+\theta_{2}\right), \\
& b_{12}=b_{21}=-l_{2} S\left(\theta_{1}+\theta_{2}\right), \quad \text { and } \\
& b_{22}=-l_{2} S\left(\theta_{1}+\theta_{2}\right)
\end{aligned}
$$

\section{A.2. 5-bar Linkage}

Let $S_{i j}^{k}=\operatorname{Sin}^{k}\left(\theta_{i}-\theta_{i i}\right)$ and $C_{i j}^{k}=\operatorname{Cos}^{k}\left(\theta_{i}-\theta_{i i}\right)$

The elements of the Jacobian, $\mathbf{J}$ of end point $P$ of 5-bar linkage (Fig. 5) are given by

$$
\begin{aligned}
& j_{11}=-l_{1} S \theta_{1}+\left(l_{1} l_{5} S_{31} / l_{2} S_{32}\right) S\left(\theta_{1}+\alpha\right), \\
& j_{12}=-\left(l_{4} l_{5} S_{34} / l_{2} S_{32}\right) S\left(\theta_{1}+\alpha\right), \\
& j_{21}=+l_{1} C \theta_{1}-\left(l_{1} l_{5} S_{31} / l_{2} S_{32}\right) C\left(\theta_{1}+\alpha\right), \\
& j_{22}=+\left(l_{4} l_{5} S_{34} / l_{2} S_{32}\right) C\left(\theta_{1}+\alpha\right),
\end{aligned}
$$


The elements of coefficient matrices, $\mathbf{A}$ and $\mathbf{B}$ are given by

$$
\begin{aligned}
& a_{11}=\left\{-l_{1} l_{2}^{3} S_{32}^{3} C \theta_{1}-l_{1}^{2} l_{2} l_{5} S_{31}^{2} C\left(\theta_{2}+\alpha\right)-l_{1}^{2} l_{2} l_{5} S_{31}^{2} C_{32} S\left(\theta_{2}+\alpha\right)-l_{1} l_{5} l_{2}^{2} S_{31}^{2} C_{31} S\left(\theta_{2}+\alpha\right)\right. \\
& \left.+l_{1}^{2} l_{3} l_{5} S_{21}^{2} S\left(\theta_{2}+\alpha\right)\right\} / l_{1}^{3} S_{32}^{3} \\
& a_{12}=a_{21}=\left\{+l_{2} l_{5} S_{31} S_{32} S_{34} C\left(\theta_{2}+\alpha\right)+l_{2} l_{5} S_{31} S_{32} S_{34} C_{32} S\left(\theta_{2}+\alpha\right)-l_{3} l_{5} S_{21} S_{24} S\left(\theta_{2}+\alpha\right)\right\} \\
& /\left(l_{1} l_{4} l_{2}^{3} S_{32}^{3}\right) \\
& a_{22}=\left\{-l_{2} l_{4}^{2} l_{5} S_{32} S_{34}^{2} C\left(\theta_{2}+\alpha\right)+l_{2}^{2} l_{4} l_{5} S_{32}^{2} C_{34} S\left(\theta_{2}+\alpha\right)-l_{2} l_{4}^{2} l_{5} S_{34}^{2} C_{32} S\left(\theta_{2}+\alpha\right)\right. \\
& \left.+l_{3} l_{4}^{2} l_{5} S_{24}^{2} S\left(\theta_{2}+\alpha\right)\right\} / l_{2}^{3} S_{32}^{3} \\
& b_{11}=\left\{-l_{1} l_{2}^{3} S_{32}^{3} S \theta_{1}-l_{1}^{2} l_{2} l_{5} S_{31}^{2} S_{32} S\left(\theta_{2}+\alpha\right)+l_{1} l_{2}^{2} l_{5} S_{32}^{2} C_{31} C\left(\theta_{2}+\alpha\right)+l_{1}^{2} l_{2} l_{5} S_{31}^{2} C_{32} C\left(\theta_{2}+\alpha\right)\right. \\
& \left.-l_{1}^{2} l_{3} l_{5} S_{21}^{2} C\left(\theta_{2}+\alpha\right)\right\} / l_{2}^{3} S_{32}^{3} \\
& b_{12}=b_{21}-\left\{l_{2} l_{5} S_{31} S_{32} S_{34} S\left(\theta_{2}+\alpha\right)-l_{2} l_{5} S_{31} S_{34} C_{32} C\left(\theta_{2}+\alpha\right)+l_{3} l_{5} S_{21} S_{24} C\left(\theta_{2}+\alpha\right)\right\} \\
& /\left(l_{1} l_{4} / l_{2}^{3} S_{32}^{3}\right) \\
& b_{22}=\left\{-l_{2} l_{4}^{2} l_{5} S_{32} S_{34}^{2} S\left(\theta_{2}+\alpha\right)-l_{2}^{2} l_{4} l_{5} S_{32}^{2} C_{34} C\left(\theta_{2}+\alpha\right)-l_{2} l_{4}^{2} l_{5} S_{34}^{2} C_{32} C\left(\theta_{2}+\alpha\right)\right. \\
& \left.-l_{3} l_{4}^{2} l_{5} S_{24}^{2} C\left(\theta_{2}+\alpha\right)\right\} / l_{2}^{3} S_{32}^{3}
\end{aligned}
$$

\section{References}

[1] B. Roth, Performance evaluation of manipulators from a kinematic view point, National Bureau of Standards Workshop on Performance Evaluation of Manipulators, 1970.

[2] A. Kumar, K.J. Waldron, The workspaces of a mechanical manipulator, ASME J. Mech. Des. 103 (July) (1981) 665-672.

[3] Bajpai, B. Roth, Workspace and mobility of a closed loop manipulator, Int. J. Rob. Res. 5 (2) (1986) 131-142.

[4] J.K Salisbury, B. Roth, Kinematic and force analysis of articulated mechanical hands, Trans. ASME J. Mech. Transm. Autom. Des. (March) (1983) 35-41.

[5] H. Asada, I.H. Ro, A linkage design for direct drive arms, ASME J. Mech. Transm. Autom. Des. 107 (1985) 536540 .

[6] T. Yashikawa, Manipulability of robotic mechanisms, Int. J. Rob. Res. 4 (2) (1985) 3-9.

[7] A. Ghosal, B. Roth, Instantaneous properties of multi-degrees-of-freedom motions-point trajectories, ASME J. Mech. Transm. Autom. Des. 109 (1987) 107-115.

[8] M.V. Lee, A.G. Erdman, Y. Gutman, Kinematic/kinetic performance analysis and synthesis measures of multidegree-of-freedom mechanisms, Mech. Mach. Theory 28 (5) (1993) 651-670.

[9] D.R. Howe et al., Grasping manipulation and control with tactile sensing, CDR Technical Report No. 891206, Stanford University, Stanford, CA, 1989.

[10] Y. Ou, L.W. Tsai, Kinematic synthesis of tendon-driven manipulators with isotropic transmission characteristics, ASME J. Mech. Des. 115 (December) (1993) 665-672.

[11] C. Gosselin, J. Angeles, A new performance index for the kinematic optimization of robotic manipulators, ASME Trends Develop. Mech. Mach. Rob. DE-15 (3) (1988) 441-447.

[12] G. Strang, Linear Algebra and its Application, Academic Press, New York, 1976.

[13] K.N. Umesh, Studies on instantaneous kinematic properties of planar two-degrees-of-freedom robotic linkages, Ph.D. Dissertation, Indian Institute of Technology, Bombay (India). 